\title{
Radial deformation measurement of a cylinder under compression using multicore fibre
}

\author{
Amanda Fender*a, William N. MacPherson ${ }^{\mathrm{a}}$, Robert R. J. Maier ${ }^{\mathrm{a}}$, James S. Barton ${ }^{\mathrm{a}}$, \\ Julian D.C. Jones ${ }^{\mathrm{a}}$, Kevin S. Ellis ${ }^{\mathrm{b}}$, Clare L. Leppard ${ }^{\mathrm{b}}$, Paul G. Blackwell ${ }^{\mathrm{b}}$, James R. Miller ${ }^{\mathrm{b}}$, \\ Benjamin J. S. Jones ${ }^{\mathrm{b}}$, Scott McCulloch ${ }^{\mathrm{b}}$, Xianfeng $\mathrm{Chen}^{\mathrm{c}}$, Rui Suo ${ }^{\mathrm{c}}$, Lin Zhang ${ }^{\mathrm{c}}$ \\ (a) Applied Optics and Photonics, School of Engineering \& Physical Sciences, Heriot-Watt \\ University, Edinburgh, EH14 4AS; (b) AWE Plc, Reading, RG7 4PR, UK; (c) Photonics Research \\ Group, School of Engineering \& Applied Science, Aston University, Birmingham B4 7ET, UK
}

\begin{abstract}
A multicore fibre (MCF) sensor to measure the radial deformation of a compliant cylinder under compression is presented. The sensor is connectorised and need not be permanently bonded to the test object. A differential measurement technique using FBGs written into the MCF makes the sensor temperature insensitive. FBG measurement of axial strain of a cylinder under compression is also reported.
\end{abstract}

Keywords: Fibre Bragg gratings, multicore, radial deformation, compression, curvature

\section{INTRODUCTION}

We have previously reported two-dimensional curvature measurement using multicore fibre (MCF) for both dynamic ${ }^{1}$ and quasi-static ${ }^{2}$ testing. Our technique uses a $125 \mu \mathrm{m}$ diameter silica fibre with four cores arranged in a $50 \mu \mathrm{m}$ square (Fig. 1). A fibre Bragg grating (FBG) is written into each core at approximately the same wavelength at the same axial position in the fibre (Fig. 2). When the fibre is bent, each of the four gratings will be stretched or compressed, depending on its position relative to the bend axis. By taking a differential measurement between pairs of cores, the curvature, $\kappa$, of the fibre may be recovered as $\kappa=\Delta \varepsilon / \mathrm{d}$, where $\mathrm{d}$ and $\Delta \varepsilon$ are the distance and strain difference between the two cores respectively. The differential measurement method makes the MCF bend sensor insensitive to common-mode effects such as temperature and axial strain. A further advantage of this sensing technique is the sensor need not be permanently bonded to the test object since net axial strain does not need to be transferred to the FBGs. This is important for soft test materials. However, the MCF must accurately take up the shape of the test piece to provide a meaningful curvature result.

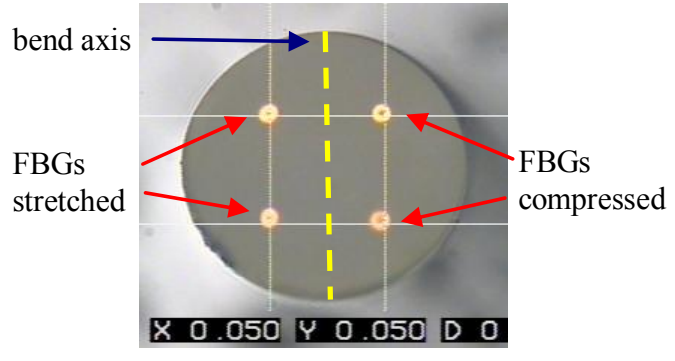

Fig. 1. Cross section of four-core fibre.

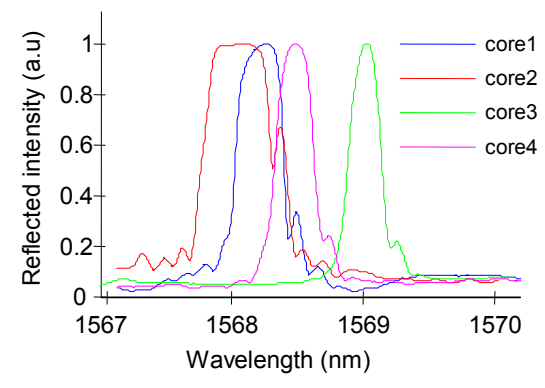

Fig. 2. Reflection spectra of FBGs written into MCF

In this paper, we present our MCF curvature sensing technique applied to the measurement of the radial deformation of a compliant cylinder (10.2 mm radius polypropylene) under compression. We envisage that this technique may be applied to test objects made of materials to which fibres may not be easily bonded. This method may be used in hazardous environments with restricted optical access, where electrical sensors ${ }^{3}$ and full-field optical methods ${ }^{4}$ may not be suitable. We also report FBG measurement of the axial strain of the cylinder during compression.

19th International Conference on Optical Fibre Sensors, edited by David Sampson, Stephen Collins, Kyunghwan Oh, Ryozo Yamauchi, Proc. of SPIE Vol. 7004, 70040G, (2008) 0277-786X/08/\$18 doi: 10.1117/12.785820 


\section{RADIAL DEFORMATION MEASUREMENT USING MCF FBGS}

\subsection{Sensor preparation}

The position of each individual MCF core was found by illuminating the grating with a visible laser and using a microscope to locate the position and height of the light scattered from the core. In this way, the fibre was orientated as shown in Fig. 1, so that no cores lie on the neutral axis of the bent fibre. A MCF connector ${ }^{5}$ was spliced to the MCF FBGs. Since the interrogation system was also connectorised, this made the MCF curvature sensors easily interchangeable.

To simplify the attachment process, the MCF FBGs were embedded in a compliant material. Since the fibre needs only to take up the shape of the test piece and does not need to undergo axial strain, it may be wrapped around the cylinder and held at one point with limited slippage possible to accommodate test object shape change. For these tests, the MCF gratings were embedded inside a rectangular cross section strip of 'Sylgard' $\sim 100 \times 5 \times 2 \mathrm{~mm}$. Care was taken to ensure that the fibre was positioned at the bottom of the strip so that the FBGs were placed close to the test piece and prevent any curvature change being absorbed by the Sylgard.

\subsection{Calibration of sensor}

To calibrate the sensor, the Sylgard strip was placed into curved grooves of known radii machined into a plate and the FBG spectra were recorded using a Smartfibres W4 interrogator in the arrangement shown in Fig. 3. The difference between two FBGs which would be on opposing sides of the neutral axis when the fibre was bent is plotted as a function of fibre curvature in Fig. 4.

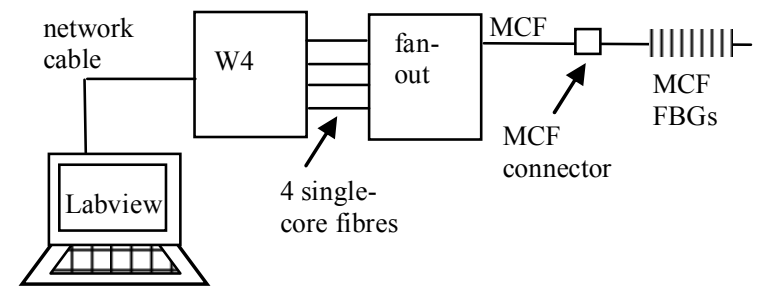

Fig. 3. Interrogation system

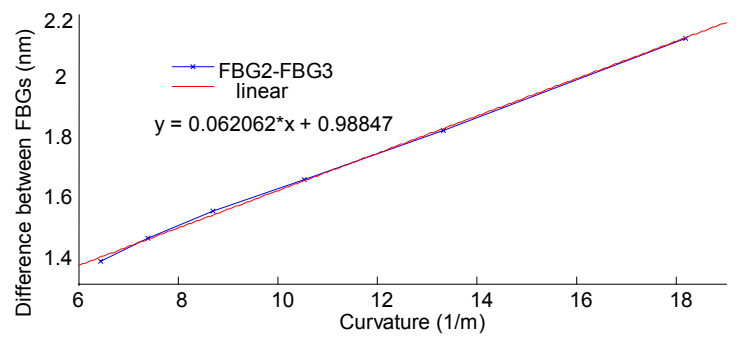

Fig. 4. Difference between FBGs located at opposite sides of the neutral axis of the MCF for various bend radii

The equation of a linear fit to this plot results in a FBG sensitivity of $\sim 1.2 \mathrm{pm} / \mu \varepsilon$. The standard deviation of the difference between the data and the linear fit is $\sim 9 \mathrm{pm}$. Taking twice this value gives an error in the difference of FBG shifts of $18 \mathrm{pm}$. This translates to an error in curvature of $0.3 \mathrm{~m}^{-1}$. For our test samples with $10.2 \mathrm{~mm}$ radius this relates to a radius measurement error of $\pm 30 \mu \mathrm{m}$.

\subsection{Compression testing}

To attach the MCF to the polypropylene cylinder (10.2 mm radius, $50.8 \mathrm{~mm}$ length), the Sylgard strip was wrapped around and held in place with a cable tie to ensure that the MCF FBGs took the shape of the cylinder, whilst allowing for some slippage as the radius increased during compression. The FBGs spectral profiles were all found to broaden by $\sim 20 \%$ and change their overall shape slightly due to the small bend radius of the test cylinder. This was not an important effect for these tests since the change in cylinder radius during deformation was small and hence, the profile would not change significantly during the test.

Fig. 5 shows the cylinder instrumented with the MCF and linearly variable differential transformers (LVDTs) to provide a reference measurement. A gap of $\sim 1 \mathrm{~cm}$ was left between the Sylgard and the LVDTs so that they did not come into contact during compression.

The cylinder was compressed using a hydraulic compressor (Denison T42B3). The shifts of the sensing MCF FBGs were converted to a value for the cylinder radius using the calibration from Fig 4. The measured radius is plotted as a function of compressive force in Fig. 6. The radius at $\sim 0 \mathrm{kN}$ compressive force is $10.24 \mathrm{~mm}$, which is slightly larger than the specified radius as a small pre-force was applied to hold the sample in place before the main loading cycle. 


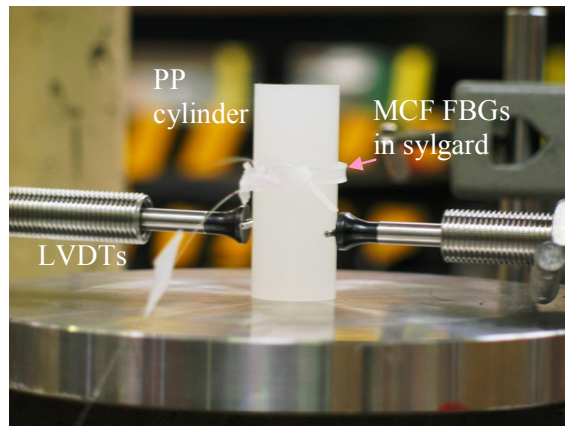

Fig. 5. Cylinder instrumented for radial measurement before compression

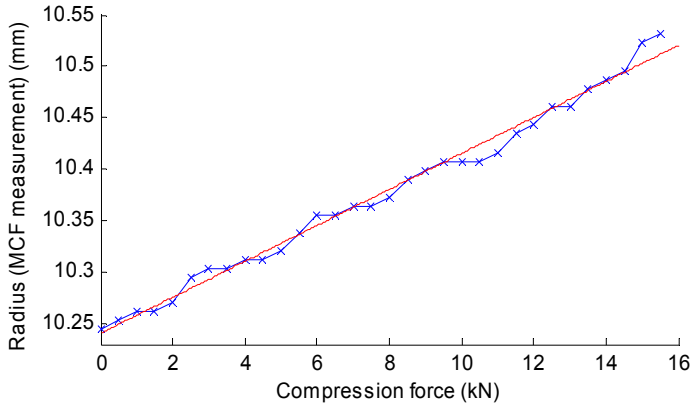

Fig. 6. Measured change in cylinder radius as a function of compression force

The cylinder radius measured by the MCF is plotted against the radius measured by the LVDTs in Fig. 7. A linear fit to this plot has a gradient of $1.2 \mathrm{~mm} / \mathrm{mm}$. This deviation from unity can be explained by the fact that the LVDTs and MCF were measuring the cylinder's radius at different axial locations. After relaxation, the diameter changes, measured with callipers, at the MCF and LVDT locations were $\sim 0.51 \mathrm{~mm}$ and $\sim 0.40 \mathrm{~mm}$ respectively, or a ratio of 1.28 , in agreement with the slope in Fig.7. The standard deviation of the difference between the linear fit to this plot and the data is 17 microns.

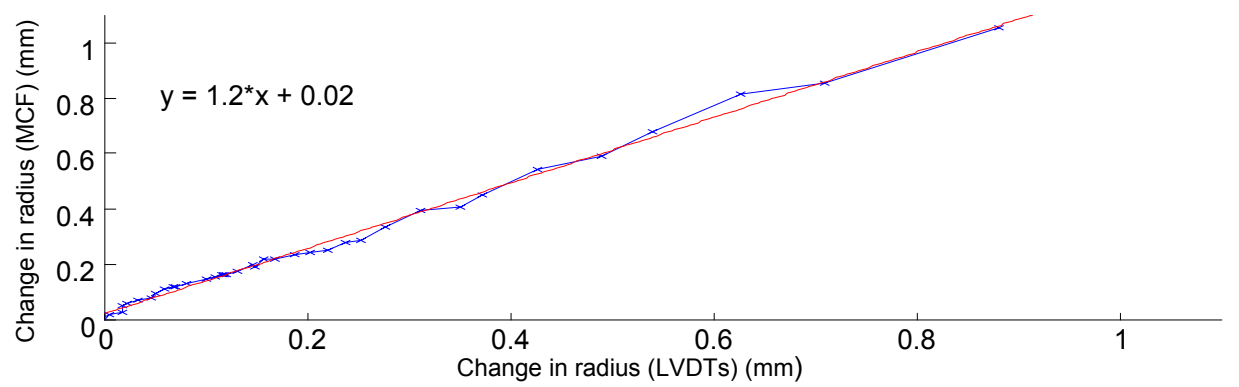

Fig. 7. Comparison between cylinder radius measured at LVDT location and MCF location

\section{AXIAL COMPRESSION MEASUREMENT USING A FBG}

\subsection{Sensor preparation}

For axial strain measurement (not curvature) a single core fibre incorporating a FBG was used. The FBG was prestrained to shift the FBG by $\sim 0.7 \mathrm{~nm}$, equivalent to approximately $700 \mu \varepsilon$. Vertical pre-straining ensured that the fibre was attached parallel to the vertical axis of the cylinder (axis of compression). The surface of the cylinder was made slightly rough by rubbing with sandpaper to provide a better surface for the epoxy (Araldite) to fix the pre-strained fibre to the test-piece. The fibre was adhered along the length of the grating to prevent bowing during compression. No change in the profile of the reflection spectrum was apparent after attachment.

\subsection{Compression testing}

The polypropylene cylinder instrumented with the pre-strained FBG was inserted between the plates of the compression machine and a pre-force was applied to hold the sample in place. A second FBG mounted strain-free in a stainless steel tube was placed close to the cylinder to monitor temperature. The FBGs were interrogated with the Smartfibres W4 interrogator as before.

Fig. 8 shows the shifts of the FBGs for temperature and strain measurement and the change in length of the cylinder (compression distance) during the compression test. Before compression, to $\sim 650$ seconds, neither FBG is shifted. During compression the temperature sensing FBG signal remained constant. The strain sensing FBG was shifted $\sim 16 \mathrm{~nm}$ due to a compression of $\sim 1.2 \mathrm{~mm}$ before the fibre detached form the test sample. The strain sensing FBG spectrum was found to broaden slightly with compression and just before the epoxy delaminated, peak-splitting was evident. 


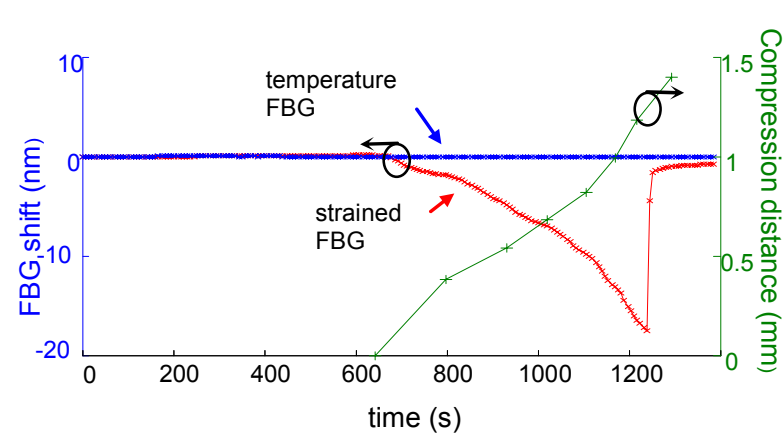

Fig. 8. Compression distance and shifts of FBGs measuring temperature and strain during compression test

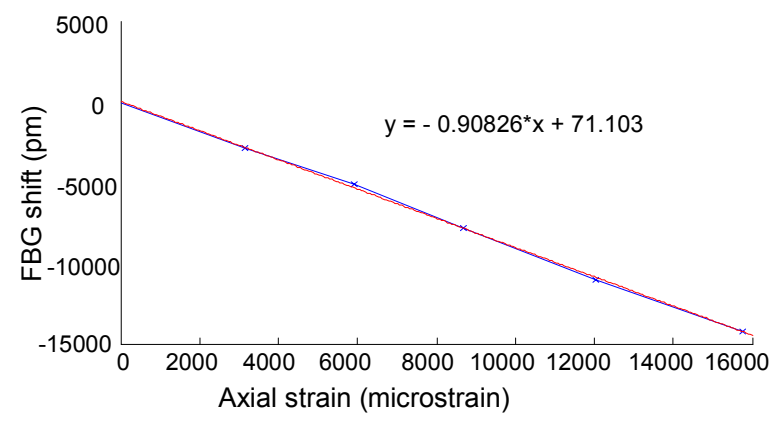

Fig. 9. FBG shift as a function of compressive strain of polypropylene cylinder

For purposes of calibration a reference measurement of the cylinder strain was determined by dividing the change in cylinder length by the initial cylinder length. The error in this reference is estimated as $\pm 200 \mu \varepsilon$ due to $50 \mu \mathrm{m}$ measurement error in change in cylinder length during compression. Figure 9 plots the shift of the FBG reflection peak before delaminating against this strain. By applying a linear fit to this plot, we find that the sensitivity of the grating is $0.9 \mathrm{pm} / \mu \varepsilon$. The standard deviation of the difference between the data points and the linear fit is $139 \mathrm{pm}$ giving a $95 \%$ confidence error of $\sim 300 \mu \varepsilon$. This value includes the error in the reference strain measurement $(200 \mu \varepsilon$, equivalent to $\sim 180 \mathrm{pm}$ ) due to measurement resolution, cylinder barrelling and spatial averaging of the strain in the reference measurement. An estimation of the FBG resolution is given by the error in FBG peak wavelength measurement, estimated as $8 \mathrm{pm}$, which indicates a FBG strain error of $7.2 \mu \varepsilon$.

\section{CONCLUSIONS}

Optical fibre techniques to measure axial strain and the change in radius of a polypropylene cylinder under compression have been described. The radial technique was demonstrated over a $1 \mathrm{~mm}$ change in radius, although this is not the limit of the system. It is difficult to place an error on this measurement since no reliable reference measurement was available, but the calibration of this sensor suggests an error in curvature of $0.3 \mathrm{~m}^{-1}$. For our test samples with $10.2 \mathrm{~mm}$ radius this relates to a radius measurement error of $\pm 30 \mu \mathrm{m}$.

Acknowledgements: The authors acknowledge the UK Engineering and Physical Sciences Research Council (EPSRC) and AWE plc for supporting this work.

\section{REFERENCES}

1 A. Fender, E. J. Rigg, R. R. J. Maier, W. N. MacPherson, J. S. Barton, A. J. Moore, J. D. C. Jones, D. Zhao, L. Zhang, I. Bennion, S. McCulloch, and B. J. S. Jones, " Dynamic two-axis curvature measurement using multicore fiber Bragg gratings interrogated by arrayed waveguide gratings," Appl. Opt., 45, 9041-9048 (2006)

2 G. M. H. Flockhart, W. N. MacPherson, J. S. Barton, J. D. C. Jones, L. Zhang, I. Bennion , "Two-axis bend measurement with Bragg gratings in multicore optical fiber," Opt Lett, 28, 387-389 (2003)

3 K. Ellis, C. Leppard, H. Radesk, "Mechanical properties and damage evaluation of a UK PBX," J. Materials Sci, 40, 6241-6248 (2005)

4 F. Chen, G.M. Brown, M. Song, "Overview of three-dimensional shape measurement using optical methods," Opt Eng, 39 (2000)

5 M. Silva-Lopez, C. Li, W. N. MacPherson, A. J. Moore, J. S. Barton, J. D.C. Jones, D. Zhao, L. Zhang, I. Bennion "Transverse load and orientation measurement using multicore fiber Bragg gratings," Appl. Opt 44, 6890 - 6897 (2005) 